\title{
The effect of 5- and 14-day intertrial intervals on the 1-Hz suppression effect
}

\author{
JOHN GAITO \\ York University, Downsview, Ontario, Canada
}

\begin{abstract}
Experiments were conducted to evaluate the effect of 5-day and 14-day intertrial intervals of $1-\mathrm{Hz}$ brain stimulation on kindling behavior induced by $60-\mathrm{Hz}$ sine-wave stimulation. The effective threshold intensity (ETI) to elicit a kindled response with $60-\mathrm{Hz}$ stimulation was determined on two separate occasions with 15 brain-stimulation trials between determinations. In Experiment 1, experimental rats were stimulated with $1-\mathrm{Hz}$ sine waves before and after a 60-Hz brain-stimulation trial, with a 14-day interval between each stimulation session (Group 160-1). A second group (Group X-60-X) received only the 60-Hz stimulation on the same trials as those on which Group 1-60-1 was stimulated with $60-\mathrm{Hz}$ current. Experiment 2 was similar to Experiment 1, but the interval was 5 days. In previous experiments with 1, 3, or $24 \mathrm{~h}$ between trials, Group 1-60-1 had had a gradual increase in the intensity required to elicit a kindled response with $60-\mathrm{Hz}$ current from $\mathrm{ETI}_{1}$ to $\mathrm{ETI}_{4}$ (the suppression effect). However, with a 72-h interval, a decline similar to that of Group X-60-X occurred in ETI values. The results with 14-day and 5-day intervals were similar to those with a 72-h interval. Suppression of kindled behavior on daily trials was not present in Group 1-60-1, in contrast to the results with 1, 3, and $24 \mathrm{~h}$. Apparently, intervals of 3 days or greater allow much of the suppression effect to dissipate.
\end{abstract}

The kindling effect has been investigated in a number of laboratories (e.g., Goddard, McIntyre, \& Leech, 1969). In rats, this effect involves a change from normal exploration (Stage 1) to behavioral automatisms (Stage 2, BA-chewing, eye closure on ipsilateral side, salivation), and finally to clonic convulsions (Stage 3, CC) in response to electrical stimulation of a specific brain site (e.g., amygdala). During Stage 3, the rat stands on its hind paws and bilateral convulsions of the forelimbs occur. A kindling progression also occurs in other animals, namely, frog, reptile, mouse, rabbit, cat, monkey, baboon (Racine, 1978). During kindling, a permanent change that does not damage tissue is assumed to occur in the brain. Behavioral, chemical, electrophysiological, and neurological aspects of this effect have been investigated by many researchers (Gaito, 1976; Racine, 1978).

In a series of experiments, it was found that $1-$ or $3-\mathrm{Hz}$ sine-wave stimulation trials suppressed the tendency of the $60-\mathrm{Hz}$ current to produce kindling behavior (e.g., Gaito, 1980a). The experiments with $3-\mathrm{Hz}$ stimulation were conducted at an intertrial interval of $1 \mathrm{~h}$ between the imposition of the 3 - and the $60-\mathrm{Hz}$ stimulation trials. Other intertrial intervals have been used with the $1 \cdot \mathrm{Hz}$ agent. The typical sequence has been one $60-\mathrm{Hz}$ stimulation trial sandwiched between $1-\mathrm{Hz}$ trials, that is, 1-60-1, usually with one sequence per day. With 1 - and 3-h intertrial intervals, the suppression effect was pronounced. The effect was present with a $24-\mathrm{h}$ interval, but was re-

The author's mailing address is: Department of Psychology, York University, Downsview, Ontario, Canada M3J 1P3. duced greatly (Gaito \& Gaito, 1981). When the interval was increased to $72 \mathrm{~h}$, the effect had dissipated (or nearly so) (Gaito, 1982). Thus, there appears to be a decay of the effect over time.

The present experiments were concerned with this process of decay over time. We were attempting to determine the specific time interval at which the effect might disappear.

\section{METHODS}

In Experiment 1, 28 male Wistar rats (about 90 days of age) had nichrome bipolar electrodes placed unilaterally in the amygdala. The brain coordinates for electrode implantation were the same as in many experiments in our laboratory: $.5 \mathrm{~mm}$ posterior to bregma, $4.5 \mathrm{~mm}$ from midline, $8.5 \mathrm{~mm}$ from skull (bregma as zero reference point).

Stimulation was not imposed until at least 7 days after surgery. Then the 28 rats were stimulated with $60-\mathrm{Hz}$ sine waves for $30 \mathrm{sec}$ during three trials on Day 1 . One hour intervened between stimulation trials. A Lafayette stimulator was used; the intensity was 36 microA (root mean square, RMS; equivalent to 100 microA, peak to peak). On the first trial of Day 2, the first effective threshold intensity $\left(\mathrm{ETI}_{1}\right)$ was determined. The $60-\mathrm{Hz}$ current was increased until a Stage 2 or Stage 3 response was elicited. Then, 5 microA was added to allow for day-to-day threshold fluctuations. Two further trials of stimulation at this intensity were provided to check on the stability of the response. The 28 rats were separated into two groups, by pairing most rats in such a way as to maintain approximately equal mean ETI values for the two groups.

Then one group of 14 rats received stimulation with $1-\mathrm{Hz}$ sine waves for $120 \mathrm{sec}$ on Trials 1 and 3,4 and 6,7 and 9, 10 and 12 , and 13 and 15 at twice the ETI $_{1}$ value. A $60-\mathrm{Hz}$ stimulation trial was provided on Trials $2,5,8,11$, and 14 for $30 \mathrm{sec}$ at $\mathrm{ETI}_{1}$ (Group 1, 1-60-1). This procedure involved a $60-\mathrm{Hz}$ stimulation trial sandwiched between $1-\mathrm{Hz}$ stimulation 
trials. There were 14 days between stimulation trials. A second group of 14 rats received $60-\mathrm{Hz}$ stimulation on the same trials as Group 1-60-1, but, on the other trials, each rat was placed in the apparatus without being stimulated (Group 2, X-60-X). All $60-\mathrm{Hz}$ stimulation was at $\mathrm{ETI}_{1}$ for $30 \mathrm{sec}$, a duration that has been used routinely in our research. Stimulation with $1-\mathrm{Hz}$ current was for $120 \mathrm{sec}$ at two times $\mathrm{ETI}_{1}$; in previous experiments, this duration and this intensity have been found to produce a drastic suppressive effect. After these 15 trials, rats from all groups had $\mathrm{ETI}_{2}$ determined over six trials during 2 days ( 3 trials/day).

At the end of Experiment 1, 14 rats remained. Of these 14, 6 were assigned to Group 1-60-1 and 4 to Group X-60-X, and Experiment 2 was conducted. The procedures were the same as before, except that the intertrial interval was 5 days. Four rats from each group completed Experiment 2.

\section{RESULTS}

In previous experiments, one dependent variable (ETI) has been most sensitive to the effects of 1 - and $3-\mathrm{Hz}$ stimulation. This variable was used in the present experiments. The ETI results for Experiments 1 and 2 are shown in Table 1. For both intervals, the ETI results do not show the typical suppression effect for the 1-60-1 groups, that is, do not show increasing ETI values over the ETI determinations; all groups evidenced the decline in threshold values typical of X-60-X groups.

Prior to Experiment 1, spontaneous convulsions had not been observed (possibly because we had not been looking for them). However, in Experiment 1, spontaneous convulsions were seen occasionally in both groups.

Both groups had an interval of 42 days ( 3 trials x 14 days) between $60-\mathrm{Hz}$ stimulation trials. The results were typical of those observed in previous experiments with long periods between stimulation (Gaito, 1978); specifically, the convulsions were more severe, the duration of the convulsions increased, and half of the rats in each group (7 of 14) lost their electrode assemblies, probably as the result of hyperactivity in the home cage.

In Experiment 2, with 5-day intertrial intervals, the durations of convulsions were as great as in Experiment 1 ; however, the seizures appeared to be less severe, fewer rats lost their electrode assemblies (only 2 of 10 rats, compared with 14 of 28 rats in Experiment 1), the rats were easier to handle, and no spontaneous convulsions were observed (within the period of approximately $1 \mathrm{~h}$ after stimulation). These rats were easier to handle

Table 1

Mean ETI in Experiments 1 and 2

\begin{tabular}{lcccc}
\hline \multicolumn{5}{c}{ ETI Determinations } \\
\hline Group & $\mathrm{n}$ & $1^{*}$ & $2 *$ & $\mathrm{ETI}_{2} / \mathrm{ETI}_{1}$ \\
\hline \multicolumn{5}{c}{ 14-Day Interval } \\
$1-60-1$ & 7 & 95.0 & 83.6 & .88 \\
X-60-X & 7 & 81.4 & 57.9 & .71 \\
& \multicolumn{5}{c}{ 5-Day Interval } \\
$1-60-1$ & 4 & 79.2 & 59.5 & .77 \\
X-60-X & 4 & 68.8 & 46.3 & .67 \\
\hline
\end{tabular}

*In microamperes.
Table 2

$\mathrm{ETI}_{2} / \mathrm{ETI}_{1}$ Ratio for Various Intertrial Intervals

\begin{tabular}{crrrrrr}
\hline & \multicolumn{6}{c}{ Intertrials (in Hours) } \\
\cline { 2 - 7 } Group & \multicolumn{1}{c}{1} & \multicolumn{1}{c}{3} & \multicolumn{1}{c}{24} & 72 & 120 & 336 \\
\hline $1-60-1$ & 1.83 & 2.25 & 1.05 & .92 & .77 & .88 \\
X-60-X & .73 & .81 & .80 & .80 & .67 & .71 \\
\hline
\end{tabular}

Note-The ratio for $E T I_{1}$ determination was 1.00 for all intervals $\left(E T I_{1} / E T I_{1}\right)$.

than were those in Experiment 1, which seemed to be in a hyperactive state overall.

Table 2 shows a measure of change from the initial threshold determination $\left(\mathrm{ETI}_{1}\right)$ to that of $\mathrm{ETI}_{2}$ for the 14- and 5-day groups, as well as those from previous research (e.g., Gaito \& Gaito, 1981). The value of the ratio $\mathrm{ETI}_{2} / \mathrm{ETI}_{1}$ for Group X-60-X remains relatively constant over the different intervals, varying from .67 to .81 . The value for the $1-60-1$ groups is greater at the 3-h interval and reaches its lowest point (below 1.0) at 3, 5 , and 14 days. There is little difference between the two groups in this ratio at these time periods. Thus, it appears that the suppression produced by $1-\mathrm{Hz}$ stimulation is lost, or almost lost, at 3,5 , and 14 days.

\section{DISCUSSION}

These experiments showed little or no inhibitory effect of $1-\mathrm{Hz}$ brain stimulation on subsequent $60-\mathrm{Hz}$ stimulation. Thus, the usual pronounced inhibitory effect (or enhanced seizure resistance) observed with 1- and 3-h stimulation began to decay at $24 \mathrm{~h}$, and was greatly reduced, or almost nonexistent, by 3,5 , and 14 days. However, with the longer time periods, seizure-state characteristics tended to be more extreme-more severe convulsions, greater duration of convulsions, more electrode assemblies lost, hyperactivity leading to difficulty in handling, and a greater tendency for spontaneous convulsions to occur. The seizure resistance is concerned with susceptibility to a seizure, whereas seizure state describes the characteristics of the seizure state and conditions surrounding it. Thus, it is necessary to separate the seizure-resistance condition from that of seizure severity (Gaito, 1980b).

Other individuals (Cain \& Corcoran, 1979; Corcoran \& Cain, 1980) have reported that kindling was achieved with 3- and $2-\mathrm{Hz}$ stimulation. These results appear to be at variance with our results. Their work differed from our research in two important aspects, however: (1) They employed biphasic square-wave pulses of $1.0-\mathrm{msec}$ duration, whereas we employed sine-wave stimulation. (2) They needed higher intensities to achieve kindling than we did, and they reported little or no kindling with lower intensities. They used 200 microA and greater, measured from base to peak. This type of measurement would be equivalent to the RMS intensity of sine-wave stimulation. Our upper limit of stimulation was 200 microA, with most stimulation intensities well below that level. We assumed that the higher intensities (of low frequencies or of any frequencies) would have an increased probability of producing lesions. Thus, we set the 200-microA level as our upper limit.

The results of the Corcoran-Cain studies are summarized as follows: "The threshold current intensity necessary was approximately $300 \mu \mathrm{A}$ (peak pulse intensity measured baselineto-peak). Intensities much below this were completely ineffective even when administered repeatedly. Pulses of $0.1 \mathrm{msec}$ duration were also completely ineffective" (Cain \& Corcoran, 1979, p. 623).

The intensity aspect may be the more important of the dif- 
ferences. Thus, an interesting result occurs: Low intensities of stimulation produce suppression of kindling, whereas high intensities elicit kindling.

These suppression results represent one of three types of interference effects reported by individuals using the broad kindling paradigm (Gaito, 1980b). The other two are: that produced by repeated, periodic stimulation of the same site with $60-\mathrm{Hz}$ current (Mucha \& Pinel, 1977); and that involving successive stimulation of alternate homologous brain sites (e.g., Goddard et al., 1969).

The interference produced by repeated stimulation of the same site with the same frequency (Mucha \& Pinel, 1977) and that generated during suppression may be precipitated by a local electrical and/or chemical inhibitory mechanism that dissipates over time. Both approaches show data that can be interpreted in a similar manner. Seizures tend to be more severe, and the duration of each one increases, with long time intervals (approximately 4 days or more) between stimulation trials (Gaito, 1978; Mucha \& Pinel, 1977). Furthermore, rats recover completely, or almost completely, from the suppression effect after 32 days (Gaito, 1981), and an intertrial interval of 3 or more days between stimulations with $1-$ and $60-\mathrm{Hz}$ current allows the effect to disappear almost completely. These results are what would be expected if a localized inhibitory mechanism that decayed over time were operating.

The third type of interference reported in the broad kindling paradigm (viz, that observed during the alternation of stimulation from primary and secondary sites) may also be the same or similar to the other two interference effects (Gaito, 1980b) in that a time-dependent process appears to be involved. A rest of 2 weeks prior to the stimulation of the secondary brain site following primary-site stimulation enhanced the positive transfer effect usually observed (faster kindling at the secondary site). Likewise, this period of rest reduced somewhat the degree of negative transfer in latency data at the secondary site. Furthermore, the negative transfer usually observed with primary-site retest dissipated after a rest of 14 or more days following secondary-site stimulation. Also, the convulsion latencies showed small changes for groups with 14 or more days of rest, whereas those with 1 or 7 days of rest had large increases in latency (McIntyre \& Goddard, 1973). Thus, these interference effects (or at least some of them) may involve the same mechanism as the interference generated by periodic stimulation of the same site and that occurring in the suppression process.

During the kindling process, stimulation of a specific site results in specific changes in the brain-wave pattern in that site; later on, similar electrical patterns occur in the contralateral site. Likewise, stimulation of one site facilitates the development of the kindling progression in the contralateral area (Goddard et al., 1969). Thus, stimulation in one hemisphere affects the contralateral hemisphere, probably via electrical means. In a recent experiment (Gaito, research underway), however, stimulation of the contralateral site with $1-\mathrm{Hz}$ sine waves appeared to have no effect on behavioral responses elicited by $60-\mathrm{Hz}$ stimulation of the ipsilateral side (a lack of suppression). These results suggest that the suppression effect (although possibly similar in some aspects) operates via a different mechanism from that of the kindling effect and that the two differ in at least two important ways: (1) The kindling effect is a relatively permanent event, whereas much of the suppression effect appears to be transient and dissipates with time. (2) The suppression effect seems to be localized to the hemisphere stimulated and produces no behavioral transfer to the opposite hemisphere. This aspect is in sharp contrast to the kindling effect, wherein positive and negative transfer occur from the primary to the secondary site.

It seems that there are two processes responsible for the overall kindling phenomena which have been observed: (1) In both hemispheres, neural changes (probably involving synaptic facilitation) that are of a relatively permanent nature (e.g., Douglas \& Goddard, 1975); (2) local electrical and/or chemical changes that dissipate over time.

During the kindling and suppression events, it is probable that the quantities of specific chemicals vary over time. Presum- ably, norepinephrine and serotonin levels could be involved, for these chemicals have been shown to affect kindling behavior (Callaghan \& Schwark, 1979; McIntyre, 1980; Munkenbeck \& Schwark, 1982; Siegel \& Murphy, 1979). It has been suggested that blockage of inhibitory aspects facilitates the kindling process. It is possible that inhibitory chemicals increase in local areas following $1-\mathrm{Hz}$ stimulation and then gradually decrease over time. However, a pattern of excitatory and inhibitory chemicals could occur. Such patterns could handle the increase in suppression of convulsive behavior with short intertrial intervals, the gradual decrease at $24 \mathrm{~h}$, and the almost complete loss at 3, 5, and 14 days, and the tendency for increased behavioral reactivity with a 14-day intertrial interval.

The results with the suppression paradigm suggest that this technique may be a useful supplement within the broad kindling methodology, and that the two methods, utilizing other sophisticated techniques as well, might provide important information relative to how the brain functions during these automatic behaviors.

\section{REFERENCES}

Cain, D. P., \& Corcoran, M. E. (1979). Rapid kindling induced by low-frequency stimulation. Neuroscience Abstracts, 5, 623.

Callaghan, D. A., \& Schwark, W. S. (1979). Involvement of catecholamines in kindled amygdaloid convulsions in the rat. Neuropharmacology, 18, 541-545.

Corcoran, M. E., \& Cain, D. P. (1980). Kindling of seizures with low-frequency stimulation. Brain Research, 196, 262-265.

Douglas, R. M., \& Goddard, G. V. (1975). Long term potentiation of the perforant path-granule cell synapse in the rat hippocampus. Brain Research, 86, 205-215.

GaIto, J. (1976). The kindling effect as a model of epilepsy. Psychological Bulletin, 83, 1097-1109.

GAITo, J. (1978). The effect of 14-day interval on the oscillation effect during sequential alternation of unilateral amygdaloid stimulation. Bulletin of the Psychonomic Society, 12, 113-116.

Gaito, J. (1980a). Gradient of interference by various frequencies on $60-\mathrm{Hz}$ kindled behavior. Canadian Journal of Neurological Sciences, 7, 223-226.

GaIto, J. (1980b). Interference effects within the kindling paradigm. Physiological Psychology, 8, 120-125.

GaITo, J. (1981). The effect of low frequency and direct current stimulation on the kindling phenomenon in rats. Canadian Journal of Neurological Sciences, 8, 249-253.

Gaito, J. (1982). The effect of a 72-h intertrial interval on the $1-\mathrm{Hz}$ suppression effect in rats. Bulletin of the Psychonomic Society, 19, 362-364.

Gaito, J., \& Gaito, S. T. (1981). The effect of several intertrial intervals on the 1-Hz interference effect. Canadian Journal of Neurological Sciences, 8, 61-65.

Goddard, G. V., McIntyre, D. C., \& Leech, C. K. (1969). A permanent change in brain function resulting from daily electrical stimulation. Experimental Neurology, 25, 295-330.

MCINTYRe, D. C. (1980). Amygdala kindling in rats: Facilitation after local amygdala norepinephrine depletion with 6-hydroxydopamine. Experimental Neurology, 69, 395-407.

McINTYRe, D. C., \& GoddaRd, G. V. (1973). Transfer, interference and spontaneous recovery of convulsions kindled from the rat amygdala. Electroencephalography and Clinical Neurophysiology, 35, 533-543.

Mucha, R. F., \& Pinel, J. P. J. (1977). Postseizure inhibition of kindled seizures. Experimental Neurology, 54, 266-282.

Munkenbeck, K. E., \& Schwark, W. S. (1982). Serotonergic mechanisms in amygdaloid-kindled seizures in the rat. Experimental Neurology, 76, 246-253.

RACINE, R. (1978). Kindling: The first decade. Neurosurgery, 3, 234-252.

Siegel, J., \& Murphy, G. J. (1979). Serotonergic inhibition of amygdala-kindled seizures in cats. Brain Research, 174, 337-340. 